\title{
Monitoring Near-Infrared Spectroscopy During Treatment with Ibuprofen to Predict Splanchnic Ischemia in a Newborn with Patent
} Ductus Arteriosus

\author{
Silvia Foligno, ${ }^{1,}{ }^{*}$ Paola Giliberti, ${ }^{1}$ Francesca Landolfo, ${ }^{1}$ Veronica Pannone, ${ }^{1}$ Pietro Bagolan, ${ }^{1}$ Andrea \\ Dotta, ${ }^{1}$ and Guglielmo Salvatori ${ }^{1}$ \\ ${ }^{1}$ Department of Medical and Surgical Neonatology, Bambino Gesu Children's Hospital, IRCCS, Rome, Italy \\ "Corresponding author: Silvia Foligno, Department of Medical and Surgical Neonatology, Bambino Gesu Children's Hospital, IRCCS, Rome, Italy. Tel: +39-668592346, Fax: \\ +39-668592893, E-mail: silviafoligno@hotmail.com
}

Received 2016 September 07; Accepted 2016 November 17.

Keywords: Near-Infrared Spectroscopy, Necrotizing Enterocolitis, Patent Ductus Arteriosus, Preterm Newborns

\section{Dear Editor,}

Necrotizing enterocolitis (NEC) is one of the most common gastrointestinal emergencies in preterm infants. Its etiology is still incompletely understood. The pathogenesis includes prematurity, alteration of innate immunity, genetic predisposition, enteral feeding, intestinal bacterial overgrowth and hypoxia/ischemia. So far, the main objective of NEC researches is to identify both non-invasive methods and biological markers to detect the disease in its early stages (1). Near-infrared spectroscopy (NIRS) could be used as a non-invasive method to measure local tissue hemoglobin oxygen saturation at both capillary and postcapillary venule. Therefore, oximetry assessed via NIRS is proposed as a 'standard of care' and it is used in neonatal intensive care units (NICUs) (2). While the cerebral district was thoroughly investigated through NIRS, only few researches were conducted on other body sites in preterm infants (3). Previous studies demonstrated that NIRS measurements in newborns with syndrome of acute abdomen might identify changes in bowel oxygenation and perfusion with lower cerebro-splanchnic NIRS ratios $(3,4)$. There is a higher risk to develop NEC in preterm newborns with patent ductus arteriosus (PDA). The use of indomethacin or ibuprofen to treat PDA in preterm infants may either decrease (through closing ductus) or increase NEC incidence (through direct constriction effect on mesenteric blood vessels). The fall of roughly $30 \%-40 \%$ of tissue oxygenation was observed in patients treated with ibuprofen or indomethacin. This decrease was more dramatic for mesenteric oxygenation than renal or cerebral values $(4,5)$. An $800 \mathrm{~g}$ female, born at $26+6$ weeks of gestational age, affected by PDA with hemodynamic significance, despite 2 courses of ibuprofen, was admitted to Bambino Gesu Children's hospital. During and after treatment with ibupro- fen, intestinal oximetry was used to disclose clinical worsening. Before considering surgical ligation, the first dose of the 3rd course of ibuprofen (10 mg/kg over 2 hours) was administered. The mesenteric regional oxygen saturation $\left(\mathrm{RSO}_{2}\right)$ was about $60 \%$, before and during the whole administration. Two hours after the end of treatment, a significant decrease was observed in $\mathrm{RSO}_{2}$ values (15\% - 20\%), without any clinical signs of intestinal hypoperfusion. Furthermore, enteral feeding was reduced to minimal enteral feeding regimen. After 12 hours, severe abdominal distention was observed. The X-ray images showed bowel distension, without pneumatosis (NEC grade I, Bell criteria) (Figure 1). Subsequently, the enteral feeding was stopped and antibiotic therapy was started (amikacin $10 \mathrm{mg} / \mathrm{kg} /$ day and ampicillin-sulbactam $75 \mathrm{mg} / \mathrm{kg} /$ day). A gradual increase in $\mathrm{RSO}_{2}$ values was observed $\left(\mathrm{RSO}_{2}\right.$ values were about $50 \%$ after 72 hours and about 60\% after 96 hours) (Figure 2); 72 hours after beginning of antibiotic therapy, the clinical and image figures went back to normal and then enteral nutrition was gradually reintroduced. This case report suggests that transcutaneous NIRS splanchnic evaluation offers the opportunity to carry out noninvasive monitoring of neonates at risk for intestinal hypoperfusion and NEC and this management could be useful in clinical practice. The literature provided evident that ibuprofen could increase the risk of NEC during PDA closure. Despite some studies, the only by Chocrane reported the efficacy and feasibility of ibuprofen to treat PDA versus indomethacin and surgical procedure, but firstly some of them were not randomized and secondly a lot of biases were in the procedure of randomization; thus, it was difficult to take stable position $(6,7)$. It is proposed to employ a supportive method to reduce the risk of NEC by a low cost, practical and feasible method to detect the reduction of blood flow 
in newborns as NIRS. Probably, the reduction of blood flow in the current case was not only a consequence of ibuprofen treatment, and the immediate realization of this possibility helped to have a quick approach. It is also known that different factors such as type of milk or amount of feeding increase the risk of NEC, but the current case suggests the possibility of using NIRS to support the treatment of closures of PDA with ibuprofen. Well-planned studies should be carried out to clarify the role of $\mathrm{RSO}_{2}$ to manage preterm newborns at risk of developing NEC.

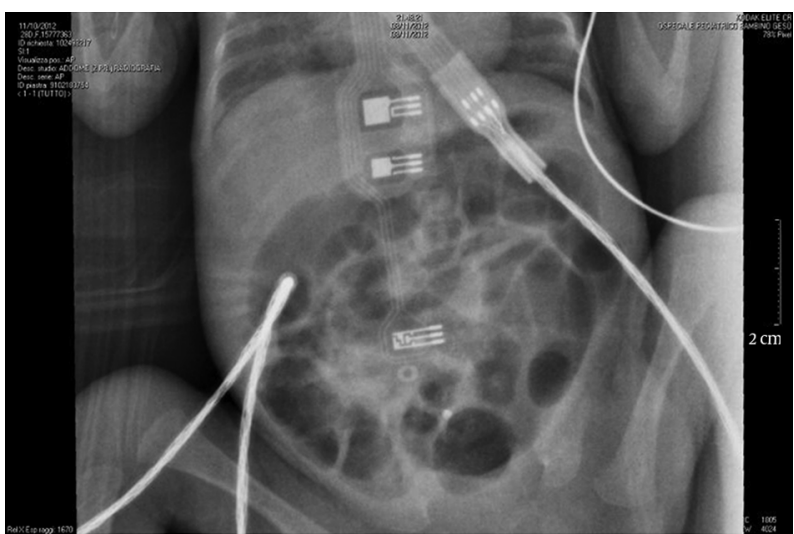

Figure 1. Abdomen X-ray Showed Bowel Distention Without Pneumatosis

Figure 2. NIRS Monitoring

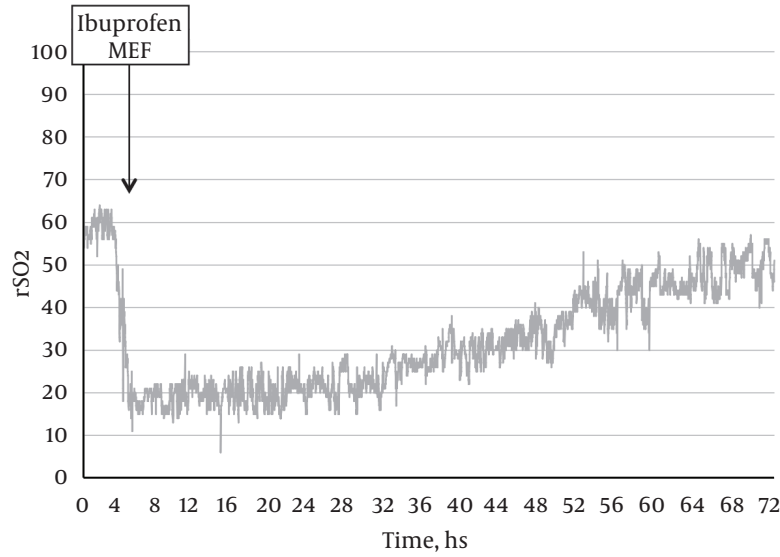

Before starting and during ibuprofen administration, the mesenterical $\mathrm{RSO}_{2}$ was about $60 \%$. At 2 hours after the end of treatment a significant (15\%-20\%) decrease was observed in $\mathrm{RSO}_{2}$ values.

\section{Footnote}

Ethical Statement: Informed consent was obtained from the parents of neonate described in the case report.

\section{References}

1. Neu J, Walker WA. Necrotizing enterocolitis. $N$ Engl $J$ Med. 2011;364(3):255-64. doi: 10.1056/NEJMra1005408. [PubMed: 21247316].

2. Cerbo RM, Cabano R, Di Comite A, Longo S, Maragliano R, Stronati M. Cerebral and somatic rSO2 in sick preterm infants.J Matern Fetal Neonatal Med. 2012;25 Suppl 4:97-100. doi: 10.3109/14767058.2012.715030. [PubMed: 22958033].

3. Gay AN, Lazar DA, Stoll B, Naik-Mathuria B, Mushin OP, Rodriguez MA, et al. Near-infrared spectroscopy measurement of abdominal tissue oxygenation is a useful indicator of intestinal blood flow and necrotizing enterocolitis in premature piglets. J Pediatr Surg. 2011;46(6):1034-40. doi: 10.1016/j.jpedsurg.2011.03.025. [PubMed: 21683194].

4. Fortune PM, Wagstaff M, Petros AJ. Cerebro-splanchnic oxygenation ratio (CSOR) using near infrared spectroscopy may be able to predict splanchnic ischaemia in neonates. Intensive Care Med. 2001;27(8):14017. [PubMed: 11511955].

5. Hirsch JC, Charpie JR, Ohye RG, Gurney JG. Near-infrared spectroscopy: what we know and what we need to know-a systematic review of the congenital heart disease literature. J Thorac Cardiovasc Surg. 2009;137(1):154-9. doi: 10.1016/j.jtcvs.2008.08.005. [PubMed: 19154918] 159e1-12.

6. Ohlsson A, Walia R, Shah SS, Ohlsson A. Ibuprofen for the treatment of patent ductus arteriosus in preterm and/or low birth weight infants. Cochrane Database Syst Rev. 2013;4:CD003481. doi: 10.1002/14651858.CD003481.pub5.

7. O'Donovan DJ, Baetiong A, Adams K, Chen A, Smith EO, Adams JM, et al. Necrotizing enterocolitis and gastrointestinal complications after indomethacin therapy and surgical ligation in premature infants with patent ductus arteriosus. J Perinatol. 2003;23(4):286-90. doi: 10.1038/sj.jp.7210911. [PubMed: 12774134]. 